$10 \mid 2018$

Devenir écrivain

\title{
Dix ans dans la vie de Kateb Yacine, de Soliloques à Nedjma
}

\section{Guy Dugas}

\section{OpenEdition}

\section{Journals}

Édition électronique

URL : https://journals.openedition.org/coma/1176

DOI : $10.4000 /$ coma. 1176

ISSN : 2275-1742

Éditeur

Institut des textes \& manuscrits modernes (ITEM)

\section{Référence électronique}

Guy Dugas, «Dix ans dans la vie de Kateb Yacine, de Soliloques à Nedjma », Continents manuscrits [En ligne], 10 | 2018, mis en ligne le 15 mars 2018, consulté le 14 janvier 2023. URL : http:// journals.openedition.org/coma/1176 ; DOI : https://doi.org/10.4000/coma.1176

Ce document a été généré automatiquement le 14 janvier 2023.

\section{(c) $(1) \&$}

Creative Commons - Attribution - Pas d'Utilisation Commerciale - Pas de Modification 4.0 International - CC BY-NC-ND 4.0

https://creativecommons.org/licenses/by-nc-nd/4.0/ 


\title{
Dix ans dans la vie de Kateb Yacine, de Soliloques à Nedjma
}

\author{
Guy Dugas
}

\section{NOTE DE L'ÉDITEUR}

Certains développements de cet article ont été publiés précédemment dans l'article "Kateb Yacine entre en littérature, d'après quelques documents inédits", de l'ouvrage collectif Littérature d'ailleurs et d'aujourd'hui. Liber amicorum offert à Ieme Ven der Poel, ss dir. Fouad laroui et Sabine Van Weselmael, éd. Zellige, 2014, pp. 73-102.

1 Kateb Yacine est né en 1929. Cette étude portera sur cinq années de son existence: 1946-1952, années au cours desquelles le jeune poète bouge beaucoup, écrit plus encore, et sur lesquelles il semble, plus tard, s'être évertué à brouiller bien des pistes. Tout commence pourtant de la manière la plus banale, s'il est possible de considérer comme banale l'expérience d'un jeune « indigène » de 17 ans désireux d'écrire dans une langue qui n'est pas la sienne. Dès cette période, l'apprenti poète songe à publier - des poèmes essentiellement: il évoque dans ses correspondances quelques pièces "groupé[es], bien plus que choisi[es $]^{1}$ " pour être soumises à la bienveillante lecture de Gabriel Audisio, à cette époque haut responsable de l'OFALAC ${ }^{2}$ et figure marquante de la vie culturelle franco-algérienne ${ }^{3}$. Et une autre lettre, presque contemporaine, à André Walter évoque des Poèmes de l'Islam réveillé, à paraître prochainement chez Seghers ${ }^{4}$ ! Au tout début de 1946, en effet, par un concours de circonstances assez extraordinaire, Kateb, qui signe encore Yacine Kateb - il n'a alors guère plus de 16 ans - était parvenu à faire imprimer à Bône quelques dizaines d'exemplaires d'un recueil de poésies, Soliloques.

\section{6-1947}

2 Peu de choses qui n'aient déjà été dites sur ce recueil, exhumé il n'y a pas si longtemps grâce à Charles Bonn, et réédité aux éditions La Découverte en 1991 : Jacqueline Arnaud 
avait placé certains de ces poèmes, ceux envers lesquels l'auteur éprouvait le moins de réticences, parmi les textes retrouvés de L'Æuuvre en fragments. Avant que le recueil tout entier, de facture très classique, ne soit retrouvé. Pour Charles Bonn, comme pour Saïd $T^{T a m b a}{ }^{5}$, c'est cette première œuvre qui, en attirant sur Kateb l'attention du gouverneur général Chataigneau, lui permit d'obtenir une bourse de séjour en France, au printemps 1947. Par quelle surprenante médiation le jeune poète parvient-il dès son arrivée à Paris à prononcer dans la salle de Sociétés savantes, qui quelques années plus tard deviendra le temple des grands débats nationalistes, une conférence sur Abdelkader et l'indépendance algérienne ${ }^{6}$ ?

3 On a énormément commenté l'intérêt de ce gouverneur envers le jeune écrivain ${ }^{7}$, où l'on a perçu tout à la fois une preuve de son intérêt pour la poésie de Kateb et une forme de sollicitude personnelle. Mais on oublie généralement de replacer ce geste dans le contexte des années d'après-guerre et surtout d'après les événements de Sétif et Guelma (8 mai 1945) - années qui virent les services du Gouvernement général tenter de rapprocher l'intelligentsia algérienne (européenne et indigène) de celle de France -, la manifestation la plus connue de cet effort ayant été les célèbres rencontres de Sidi Madani, auxquelles Kateb devait participer, ce qu'il ne fit finalement pas ${ }^{8}$. En revanche, il fait dès 1946 quelques conférences en Algérie, dont on ne sait à peu près rien, mais qui sont attestées par les correspondances ${ }^{9}$, et son nom commence à apparaitre dans quelques journaux ou revues de la colonie, comme Forge, dirigé par Emmanuel Roblès. C'est donc dans ce contexte-là - en abordant la biographie de Kateb à travers quelques documents peu connus ou inédits et quelques noms nouveaux - que je voudrais tenter de replacer la genèse de ses premiers écrits.

4 1946-1947, c'est le moment où Emmanuel Roblès, déjà fort connu en Algérie, crée en compagnie de Louis Julia et de El Boudali Safir, des «cahiers littéraires » du nom de Forge $^{10}\left(\mathrm{n}^{\circ} 1\right.$ : décembre 1946), que le comité de rédaction souhaite largement ouverts aux jeunes auteurs « indigènes ", comme on dit à l'époque. Mais ceux-ci sont alors peu nombreux - Edmond Charlot, éditeur de Camus et de Roblès, a déjà eu l'occasion de s'en rendre compte et il le déplorera souvent par la suite. Alors, lorsqu'ils veulent constituer dans le $n^{\circ} 3$ de leur revue (avril-mai 1947) un dossier sur «la jeune poésie nordafricaine ", Roblès et El Boudali battent le rappel des voix poétiques naissantes en Afrique du Nord. Ont-ils lu Soliloques? Ont-ils été mis sur cette piste par André Walter? Ou bien ont-ils été informés par quelque collaborateur de Forge de l'existence de ce jeune poète qui cherche désespérément à paraître dans le champ de la modernité culturelle de la colonie ${ }^{11}$ ? Toujours est-il que le dossier de Forge - au demeurant bien léger et dans lequel on comprend mal l'absence de tout poète tunisien ou marocain, alors même que Ahmed Sefrioui ou Salah-Eddine Tlatli ont collaboré aux deux numéros précédents - comporte, parmi des pièces de Mohammed Dib, Jean Sénac ou Ahmed Smaïli, un ami de Roblès mort accidentellement peu de temps auparavant, un poème extrait de Soliloques aux accents rimbaldiens, intitulé « Bonjour ${ }^{12}$ ».

\section{8}

5 En janvier de cette année-là, Kateb Yacine adresse à Gabriel Audisio une longue lettre dactylographiée, qui se présente comme un curriculum vitae :

Cher Ami,

À l'instant, je me souviens que vous m'aviez le jour de mon départ demandé de vous 
envoyer quelques détails biographiques.

Je suis né le 26 août 1929 à Condé Smendou (Constantine). Mon père et ma mère sont tous deux arabes de la région de Souk Ahras. Mon grand-père maternel, Si Ahmed el Ghazali Kateb, était considéré comme un des plus grands poètes de langue arabe en Afrique du Nord.

Vous pourrez trouver de ses preuves dans les anthologies éditées par les Ulémas, et dans diverses revues de Tunisie, d'Egypte et de Syrie. M. Millerand, alors Président de la République française, lui écrivit une lettre élogieuse où il lui proposait d'élever un buste à son effigie. Mon grand-père ne répondit pas à la lettre. Mes aïeux étaient tous à l'arrivée des français magistrats (Cadis) ou Ulémas. Les nécessités de l'état-civil firent que les frères dont nous descendons prirent deux noms différents. L'un donna naissance à la famille Cadi qui compta Cadi Chérif, le premier colonel d'artillerie sorti premier de Polytechnique, et l'autre s'appela Kateb (écrivain) et ses fils furent tous lettrés en arabes [sic]. Mon grand-père paternel, Kateb Salah, fut un bon grammairien, d'autres Kateb furent professeurs d'arabe, écrivains, interprètes. L'ensemble de la famille était désigné avant la domination française sous le nom de Kablout. Cadi Abdelkader n'est pas notre parent, il est d'Aïn Beïda, alors que nous sommes de Souk Ahras.

La première conseillère municipale algérienne, élue en 1945, est Zouleïkha Kateb, ma cousine ${ }^{13}$. Malheureusement, notre famille semble frappée du mal d'extinction : ces deux dernières années, elle a perdu plus de quinze de ses membres. Notre famille est très estimée, mais nous passons volontiers pour des bohèmes dénués de pratique et pour des amateurs d'alcool et de musique orientale. [Ajouté à la main : Nous sommes aussi tous pauvres et imprévoyants.]

Pour ma part, j'ai fait d'excellentes études jusqu'en quatrième. Puis j'ai négligé mes études pour lire comme un forcené et écrire. J'ai écrit mon premier poème à onze ans, c'était une satire. De onze ans à quinze ans, j'ai lu Baudelaire et Raimbaud [sic], et des livres effarants, jusqu'à Proust. Je n'ai jamais été attiré par les romantiques. Par contre, j'ai tout de suite aimé Cocteau, Eluard.

Le 8 mai 1945, j'étais encore potache, j'ai été arrêté pour atteinte à la sûreté de intérieure et extérieure de l'état, détention d'armes, participation à bandes armées, propos séditieux. Libéré quelques mois après, je me trouvais lancé dans une tournée de conférences révolutionnaires, et je fis imprimer un petit recueil intitulé Soliloques.

Pour échapper à la facilité et au ridicule de "conférencier", je suis venu à Paris, à la fois pour travailler, apprendre et changer d'air.

Comme vous le voyez, je suis un piètre biographe, mais l'essentiel était de dire ces choses... je vous salue bien, tout honteux de ce style, mais j'essaierai de me racheter en vous envoyant bientôt "L'Existence des laboureurs ${ }^{14 "}$, que je termine.

6 En avril suivant, nouvel adhérent du Parti communiste algérien en rupture avec la société coloniale, il est à nouveau en France, mais dans des conditions financières plus précaires que lors de son précédent séjour. Dès son arrivée à Marseille, il sollicite à nouveau l'aide de Gabriel Audisio, qui lui fera parvenir une somme d'argent conséquente :

Mon cher ami,

J'arrive à Marseille avec mon cousin Mohamed Tahar qui a de solides connaissances en arabe. Nous sommes véritablement acculés, n'ayant aucun moyen de gagner Paris.

Fort de vos recommandations, je suis allé voir le Dr de l'office à Marseille. C'est lui qui m'engage à vous écrire, car il n'a pas le pouvoir de nous aider.

S'il vous était possible de nous aider, mon cousin et moi, à voyager jusqu'à Paris, nous serions sauvés, car à Marseille nous ne connaissons personne. Mais au cas où cette aide vous serait impossible, je le comprendrais fort bien, surtout si cela vous devait causer du tracas. Dans ce cas, comme je suis décidé, n'importe comment à venir, je vous dis au revoir. 
Excusez-moi de vous écrire dans cette feuille entamée par la machine à écrire. Je suis chez un compatriote illettré, et emploie un commencement de manuscrit dactylographié des "Ravissements de la fête".

Bien amicalement et respectueusement ${ }^{15}$.

7 Preuve que Kateb compte toujours sur l'aide de Roblès après la disparition de Forge, il existe dans le fonds Patrimoine méditerranéen un tapuscrit de 18 pages sous chemise verte, portant, de la main de Roblès semble-t-il, mention d'un nom : «Yacine Kateb » et d'un titre : Nomades en France.

Ce texte est un fragment narratif fictionnalisant les conditions de ce pitoyable retour en France. Il met en scène Lahkdar, qui deviendra un personnage récurrent dans l'œuvre de Kateb Yacine, un double de l'auteur en bien des cas, et Larbi, prénom beaucoup moins utilisé par l'auteur de Nedjma. L'un et l'autre émigrent clandestinement vers la France à bord du Ville d'Oran. Larbi est présenté comme originaire de Djelfa, Lakhdar comme un enfant de métayers d'une ferme de soixante hectares dans la région de Sétif, fuyant la misère des campagnes algériennes en prévoyant : «Une fois payées les dettes de grand-père, je rachète les soixante hectares, je fais venir une Parisienne à cheval et je dis au colon : vous pouvez faire le vide. »

9 Après l'arrivée à Marseille, destination Arles, où Lakhdar rencontre des compatriotes qui travaillent en Camargue à l'exploitation du riz, puis parvient à se faire embaucher dans un chantier de construction. Il partage alors provisoirement la condition de manœuvre, commune à des prolétaires immigrés et français. Mais c'est Paris, et plus loin encore, que vise Lakhdar :

Depuis qu'il était à Arles, il se répétait jour et nuit qu'il était seulement à sept cents kilomètres de Paris. Du moment qu'il avait traversé la mer... Mais il n'était pas sûr d'arriver. Paris était un nuage qu'il poussait obstinément devant lui, et il savait déjà qu'il pusserait irait plus loin. Le Nord. La Belgique. Y en a qui sont allés en Amérique.

Et le personnage finit par s'embarquer dans un camion en partance vers le Nord...

Ce texte, dont l'écriture suit de peu l'épisode dont il y est question, était sans doute promis à une publication dans Forge (qui n'est plus publié à partir de fin 1947, mais... Kateb l'ignore sans doute) ou quelque autre revue projetée, comme Rivages, que Charlot rêve alors de ressusciter. Finalement, il ne sera jamais exploité par Roblès, mais se retrouvera, légèrement modifié, dans un numéro plus tardif d'Esprit ( $\mathrm{n}^{\circ} 4$, avril 1957), revue proche des Éditions du Seuil, qui contribua beaucoup à l'audience que l'œuvre de Kateb acquiert en France à partir de 1954 et la publication en ses pages du Cadavre encerclé ${ }^{16}$.

12 En cette même fin d'année 1948, cependant, une sévère mise au point de Kateb dans $L a$ République algérienne du 22 octobre (fig. 1), à la suite d'une série d'articles de Gabriel Audisio dans Combat relatifs à l'identité algérienne et à la question berbère, menace d'interrompre cette correspondance et de briser net l'amitié naissante, alors que le prêt consenti au poète par le haut fonctionnaire français est loin d'être remboursé : 
Fig. 1 : « Réponse à Gabriel Audisio », La République algérienne, 22 octobre 1948

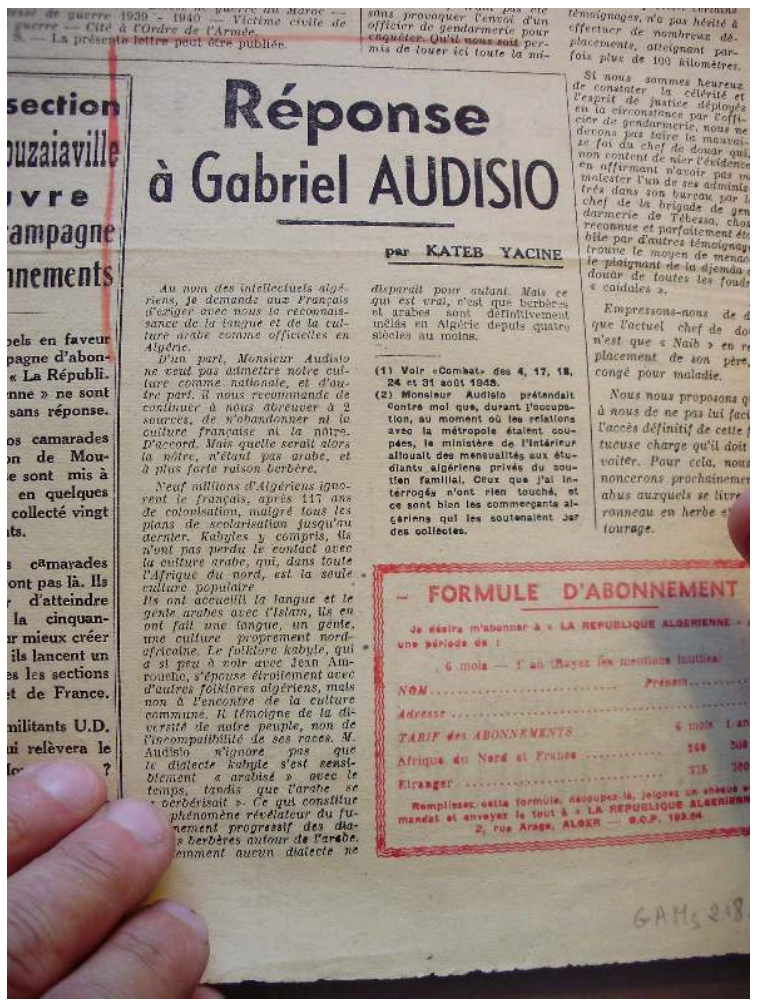

Photo : Guy Dugas

Réponse à Gabriel Audisio

Par Kateb Yacine

Au nom des intellectuels algériens, je demande aux Français d'exiger avec nous la reconnaissance de la langue et de la culture arabes comme officielles en Algérie.

D'une part, Monsieur Audisio ${ }^{17}$ ne veut pas admettre notre culture comme nationale et d'autre part, il nous recommande de continuer à nous abreuver à deux sources, de n'abandonner ni la culture française, ni la nôtre. D'accord. Mais quelle serait alors la nôtre, n'étant pas arabe, et à plus forte raison berbère?

Neuf millions d'Algériens ignorent le français, après 117 ans de colonisation, malgré tous les plans de scolarisation, jusqu'au dernier. Kabyles y compris, ils n'ont pas perdu le contact avec la culture arabe qui, dans toute l'Afrique du Nord, est la seule culture populaire.

Ils ont accueilli la langue et le génie arabe avec l'Islam. Ils en ont fait une langue, un génie, une culture proprement nord-africains. Le folklore kabyle, qui a si peu à voir avec Jean Amrouche, s'épouse étroitement avec d'autres folklores algériens, mais non à l'encontre de la culture commune. Il témoigne de la diversité de notre peuple, non de l'incompatibilité de ses races. M. Audisio n'ignore pas que le dialecte kabyle s'est sensiblement "arabisé" avec le temps, tandis que l'arabe se "berbérisait". Ce qui constitue un phénomène révélateur du fusionnement progressif des dialectes berbères autour de l'arabe. Évidemment, aucun dialecte ne disparaît pour autant. Mais ce qui est vrai, c'est que Berbères et Arabes sont définitivement mêlés en Algérie depuis quatre siècles au moins ${ }^{18}$.

Dès réception de cet article, Gabriel Audisio écrit à son jeune protégé pour se plaindre, sinon du fond de l'article, du moins de la méthode utilisée, lui rappelant qu'il n'est d'usage d'exprimer un droit de réponse que dans le journal où l'article incriminé a été publié ${ }^{19}$.

Et la réponse fuse tout aussitôt : 
Fig. 2 : Lettre de Kateb Yacine à Gabriel Audisio

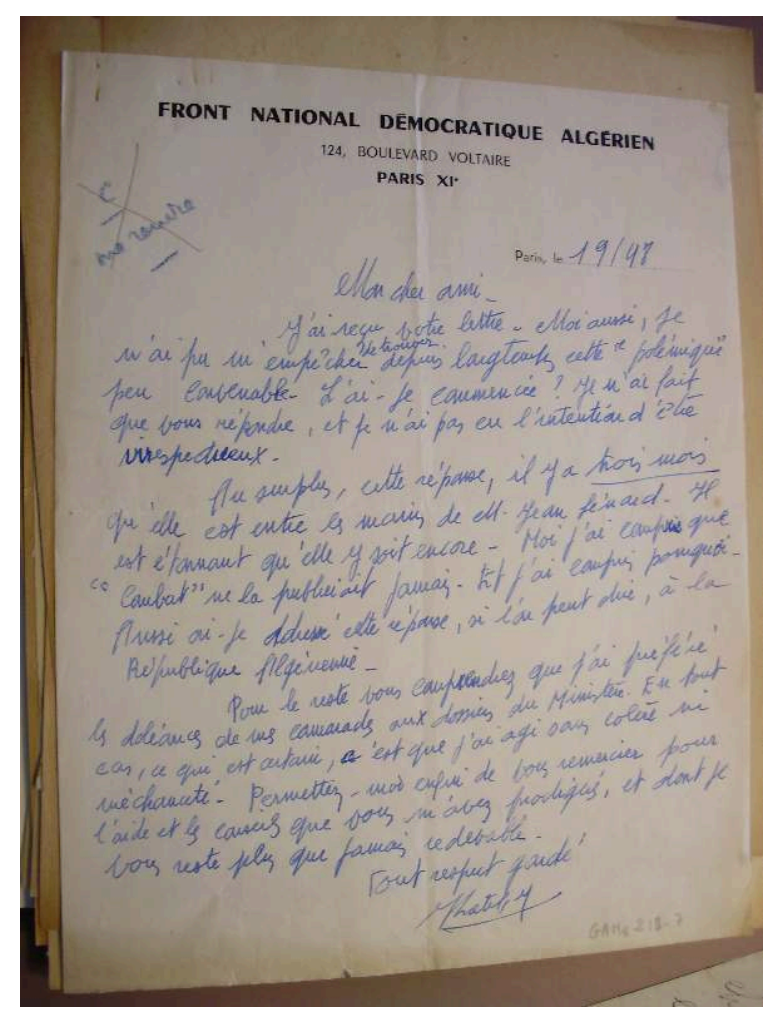

Fonds Audisio, Bibliothèque de l'Alcazar, Marseille

Paris le 19/48

Mon cher ami,

J'ai reçu votre lettre. Moi aussi, je n'ai pu m'empêcher de trouver depuis longtemps cette "polémique" peu convenable. L'ai-je commencée? Je n'ai fait que vous répondre, et je n'ai pas l'intention d'être irrespectueux.

$\mathrm{Au}$ surplus, cette réponse, il y a trois mois qu'elle est entre les mains de M. Jean Lénard. Il est étonnant qu'elle y soit encore. Moi, j'ai compris que "Combat" ne la publierait jamais. Et j'ai compris pourquoi. Aussi ai-je adressé cette réponse, si l'on peut dire, à "La République Algérienne".

Pour le reste, vous comprendrez que j'ai préféré les doléances de mes camarades aux dossiers du Ministère ${ }^{20}$. En tous les cas, ce qui est certain, c'est que j'ai agi sans colère ni méchanceté. Permettez-moi enfin de vous remercier pour l'aide et les conseils que vous m'avez prodigués, et dont je vous reste plus que jamais redevable. Tout respect gardé.

Kateb $Y^{21}$.

La menace sur l'improbable amitié qui vient de naître entre les deux hommes - l'un jeune révolutionnaire algérien, l'autre haut fonctionnaire des services coloniaux de la culture et de l'information - est d'autant plus grande qu'en ce même mois de novembre 1948, Kateb Yacine, qui se présente comme secrétaire général d'un Front national démocratique algérien, préfiguration, selon lui, du $\mathrm{FLN}^{22}$, organise et anime un meeting explosif qui lui attirera les foudres des autorités coloniales comme des messalistes. On jugera du caractère visionnaire de la conférence qu'il prononce au cours de ce meeting et dont le tapuscrit figure parmi les documents du fonds Audisio :

Oligarchie colonialiste, lutte des classes et prolétariat ${ }^{23}$

Après la mort d'Abdelkader, le Gouverneur général de l'Algérie put "gouverner"

comme il l'entendait. Ses intentions étaient loin d'être mystérieuses. Il voulait 
d'abord écraser toute velléité de résistance ou de rébellion populaire pour installer ensuite le règne de la "Chrétienté française" et pouvoir, à la place de l'économie nationale ruinée par l'incurie des Turcs et la guerre de l'Indépendance, échafauder une sorte de succursale autonome de l'impérialisme français.

Cet impérialisme trouvait terrain vierge, et propice. Épuisées par la famine et les répressions, les classes sociales de l'Algérie avaient perdu toute conscience et vivaient dans la confusion. Retenue par la pusillanimité des féodaux autochtones qui protestaient pourtant de leur patriotisme, la population ne pouvait s'opposer à l'occupation française. Elle ne voyait plus que le potentiel militaire des envahisseurs, ne pouvant discerner leur empire économique. En effet, au début de l'occupation, les propriétaires fonciers, les hobereaux, les commerçants, les représentants du culte furent brimés au même titre que paysans, artisans et nomades. L'ensemble des couches laborieuses et même un certain nombre de féodaux furent frappés par les mêmes impôts écrasants, les mêmes mesures vexatoires.

C'est ainsi que, l'asphyxie de l'économie aidant, la lutte des classes s'éteignit presque. Riches ou pauvres, paysans ou bourgeois, les Algériens s'agglomérèrent en une masse privée de vie, n'offrant plus aucune résistance à l'ennemi. Subjuguées par les rigueurs et les péripéties de l'occupation, les masses laborieuses de l'Algérie ne virent pas que nos féodaux cherchaient déjà à lier partie avec l'occupant, pour conserver leurs privilèges.

En fait, à peine la "pacification" terminée, les Français s'empressèrent de se rapprocher de ces féodaux, ainsi que des plus puissants parmi les commerçants et les Ulémas, lesquels se soumirent sans trop hésiter.

Or l'impérialisme ne pouvait définitivement s'installer dans notre pays avec le seul appui du Gouvernement général et des féodaux autochtones. La résistance du peuple algérien ne pouvait être brimée que si l'Algérie était entièrement dominée à la fois par les troupes d'occupation françaises et par un vaste réseau d'administrations et d'entreprises impérialistes largement répandues à travers les territoires.

La ruée des financiers et des négriers vers la nouvelle "colonie" nécessita par ailleurs de nouvelles constructions, tandis que le Gouvernement général recrutait dans la métropole de nouveaux effectifs militaires, une police, des "administrateurs", etc. Puis les émissaires de la Chrétienté se mirent de la partie. Ils voulaient non seulement assouvir une haine religieuse refoulée depuis les Croisades, mais aussi participer aux bénéfices... Ils ne se contentèrent nullement $d u$ rôle de missionnaires; ils se mêlèrent activement à la vie politique, et grâce à eux une véritable Citadelle Cléricale se dressa dans notre patrie ${ }^{24}$.

Alors Bugeaud et ses successeurs durent faire appel à un troisième élément "colonisateur", le plus dangereux: les propriétaires fonciers européens, à qui l'expropriation allait livrer la totalité des ressources nationales. En effet, les experts du Gouvernement général comprirent vite que l'Algérie, ayant une économie basée sur l'exploitation agricole, il ne pouvait être question de s'y établir sans installer un paysannat européen omnipotent, maître des terres, de la production agricole, et même du commerce extérieur. Nombre de financiers rechignèrent, mais le temps pressait. Le sort de l'impérialisme n'est-il pas de mourir de pléthore ? Pour rester maîtres de l'Algérie, les potentats du Gouvernement général durent se laisser envahir par la nouvelle classe exploiteuse des colons.

Ainsi naquit le colonialisme. Notre peuple dut alors se débattre sous une écrasante oligarchie à trois formes :

a) Oligarchie militaire, policière, administrative groupée autour du Gouvernement général, soumise en principe à la surveillance du Parlement français, mais se dérobant à cette surveillance et ne l'acceptant que pour la forme.

b) Oligarchie cléricale, s'acharnant contre l'âme même de notre peuple, participant à la politique impérialiste sur le plan intérieur.

c) Oligarchie foncière, composée de colons possédant la presque totalité des 
richesses agricoles et du commerce extérieur avec la Métropole, étouffant jusqu'à la féodalité autochtone ${ }^{25}$ et dirigeant sur place la politique du Gouvernement général. Pour faire oublier aux opprimés l'expropriation et la transformation des mosquées en cathédrales, pour faire croire à la prospérité de l'entreprise impérialiste, la classe exploiteuse des colons fit à son tour appel à d'autres couches de la population européenne qui lui fournirent des ouvriers qualifiés, des techniciens, des ingénieurs, des architectes, des instituteurs, des avocats, des médecins, des marins, etc.

De même, l'affluence des capitaux, l'essor du commerce entre les "colonies" et la Métropole, la croissance de l'édifice colonialiste, les besoins des nouveaux venus firent naître, bien que malgré les colons, un certain nombre de petites industries, surtout dans les villes principales. Toutefois, toutes ces industries restèrent toujours insuffisantes, par la volonté des colons qui ne les avaient tolérées que parce que leur propre existence y trouvait confort et bien-être.

C'est alors que se pose le problème de la main-d'œuvre. Malgré leurs efforts, les colons ne purent empêcher certains Algériens de travailler à titre de prolétaires salariés, aux côtés des travailleurs européens...

Or les colons s'étaient farouchement opposés à toute extension de l'Industrie, c'est qu'ils tenaient à leur sous-prolétariat agricole et ne se souciaient peu de voir se développer dans les villes une main d'œuvre autochtone salariée.

Ils considéraient les citadins comme plus "éveillés" que les paysans, donc comme plus difficiles à exploiter. Ils espéraient enfin que, maintenus dans les exploitations agricoles de la campagne, ignorant de tout droit au salaire, les paysans resteraient à leur merci.

Séparer les paysans, des ouvriers travaillant dans les petites industries des villes, pour les empêcher de s'éveiller à la conscience de classe, telle a toujours été la politique des colons.

En effet, jusqu'à la première guerre mondiale, ils "nourrissaient" les fellahs employés chez eux, mais ne leur donnaient pratiquement aucun salaire (compte non tenu des sommes dérisoires qu'ils leur distribuaient à de rares occasions, presque à titre d'aumône).

On conçoit bien après cela que les colons se soient toujours inquiétés de voir naître, aux côtés du prolétariat européen, une main-d'œuvre industrielle autochtone salariée, consciente de ses droits et de l'exploitation à laquelle étaient livrés ses frères de classe paysans.

En outre l'aménagement de routes, de ponts, de barrages dans les régions les plus reculées força le Gouvernement général à employer de nouveaux travailleurs et autochtones, créant malgré lui, et malgré les craintes des colons, un prolétariat unique où devraient entrer définitivement un nouvel élément national de l'Algérie : les exploités européens.

Il est évident que l'étouffement de la lutte des classes, la haine raciale, la propagande réactionnaire des cléricaux et les manœuvres de division des colons ont influé sur ces européens exploités, et les ont momentanément éloignés de la population laborieuse autochtone, dont ils partagent pourtant les conditions de vie. On leur fait croire qu'ils sont, eux aussi, des "civilisateurs" menacés par la révolte populaire. Mais rien ne pourra empêcher qu'ils s'éveillent bientôt à la conscience révolutionnaire et luttent aux côtés de toutes les masses laborieuses de l'Algérie, contre l'exploitation colonialiste. Déjà ils rejoignent en masse les syndicats et le Parti des ouvriers, participant à la lutte du peuple algérien, par des grèves victorieuses, des manifestations revendicatives. Il nous appartient de les entraîner encore plus nombreux au combat pour la Libération nationale, pour la conquête du pouvoir par les travailleurs révolutionnaires.

En conclusion, bien que noyée dans le combat mortel que poursuit le peuple algérien depuis plus d'un siècle, la lutte des classes devient une réalité de plus en plus vivace. Notre prolétariat s'organise dans le feu de la lutte contre 
l'impérialisme. Il nous reste à aider des millions de travailleurs agricoles et de paysans à prendre conscience de leurs intérêts, de leur destin.

Quant à la bourgeoisie algérienne, il convient de noter qu'elle est aujourd'hui dans sa majorité anti-colonialiste. En tant que telle, elle contribue en seconde place au mouvement de Libération nationale. Elle a, elle aussi, ses buts propres, qui sont forcément distincts des nôtres. Car nous ne devons jamais nous confondre avec la bourgeoisie, même si temporairement elle fait cause commune avec les exploités. Actuellement, nous avons besoin de son alliance et nous devons faire bloc avec elle pour libérer la Patrie.

Aussitôt cette étape atteinte, le devoir de tout révolutionnaire sera de barrer la route à la bourgeoisie, pour l'empêcher d'acquérir de nouveaux privilèges, à la faveur de la victoire populaire.

Il est clair qu'il ne saurait y avoir de Parti unique en Algérie. Sous la domination turque, comme sous la domination française, bourgeois et féodaux ont trahi la cause du peuple.

Aujourd'hui le peuple se lève. La situation actuelle nous commande de faire bloc avec la bourgeoisie et de l'entraîner au combat. Mais une fois la Libération nationale arrachée, soyons vigilants : les ressources et le pouvoir d'une Algérie libre et indépendante ne sauraient appartenir à d'autres qu'aux travailleurs.

\section{9}

15 On aurait pu s'attendre à ce que cette correspondance se distende un peu, à la suite de telles prises de position publiques autant que pour des raisons plus personnelles. D'autant plus que Kateb Yacine est rentré au pays, où il écrit désormais beaucoup, fournissant en textes politiques les journaux locaux, tels qu'Alger républicain, dont il est devenu un collaborateur régulier, ou Liberté, organe du Parti communiste algérien, auquel il donne "Le déshérité des Aurès ", un texte puissant et cruel sur la misère des populations rurales ${ }^{26}$.

16 Il n'en est rien encore, et la condamnation en règle de la société coloniale à laquelle Kateb se livre régulièrement n'empêche pas Gabriel Audisio de le solliciter, quelques mois plus tard, en vue de la réalisation d'un mystérieux film documentaire sur l'Algérie. Kateb répond ${ }^{27}$ :

Chers amis,

À mon sens, il faut un film sur la société algérienne moderne, plutôt que sur le peuple. Pour peindre la vie du peuple, il faudrait se placer dans sa lutte, ce qui est impossible dans les circonstances politiques actuelles. Le film serait censuré. À moins de passer sous silence l'essentiel.

Reste une chose possible, utile, et plaisante à faire: la satire de la société colonialiste. Sous cet angle, on peut atteindre en France un public assez divers pour défier la censure. Et le film sera dynamique, comique, significatif, accessible à tout le peuple de France et d'Algérie.

Scénario: Vous recommandez de ne pas envoyer de manuscrit. Je vous en adresse pourtant un, où vous trouverez, mieux que dans des phrases, mon "fonds d'idées". Quant au ciel de l'Algérie, aux civilisations orientale et occidentale, je pense que ce sont des choses sans rapport avec un film vivant.

Je souhaite que beaucoup d'Algériens participent à vos travaux. L'œuvre doit pouvoir être accomplie.

Je vous envoie mon amitié et mes modestes encouragements.

Kateb Y.

Je vous demanderai de bien vouloir me renvoyer mon manuscrit aussitôt lu, car je n'en possède plus de double. 
17 Aucun manuscrit n'accompagnant cette lettre, on peut supposer qu'Audisio, obtempérant au post-scriptum de son interlocuteur, a renvoyé le scénario joint... à moins que le scénario soit composé du texte de la conférence précédente - ce qui relèverait de la provocation!

\section{0-1951}

18 On ne relève que très peu de choses dans cette correspondance durant les deux années suivantes, même pas les habituelles cartes de vœux que Kateb avait l'habitude d'adresser à Audisio depuis trois ou quatre ans. Kateb est en Algérie, à présent chargé d'une famille de cinq personnes après la mort de son père (1950). Tout en exerçant divers métiers manuels, il continue à écrire, non seulement dans la presse algérienne, mais également son roman, dont il ne tardera pas à communiquer le brouillon à son correspondant, sollicitant son approbation et son appui auprès d'un éditeur.

19 Mais toujours pas de rencontre, bien que, semble-t-il, Kateb ait passé l'été 1951 à Paris $^{28}$. Car il y a cette dette d'amitié que le jeune poète ne peut rembourser et qui empoisonne leur relation, au moins dans l'esprit de celui-ci. Et ce n'est que fin 1951 que Kateb Yacine, "estim[ant] en fin de compte qu'il est capable de trop de silence ", osera reprendre la relation épistolaire, effaçant de lui-même la dette au bénéfice d'une hypothétique communion littéraire: "Comprenez-moi et soyons bientôt unis pour une littérature algérienne nationale et progressiste ${ }^{29}$.»

\section{2}

Arrive 1952, année capitale dans l'accomplissement de la carrière d'écrivain de Kateb Yacine. Au printemps, et après plusieurs mois de mutisme envers lui, c'est encore à Gabriel Audisio qu'il confie la lecture d'un ensemble de textes volumineux et disparates, "matériaux d'un premier roman qui ne sera pas achevé avant plusieurs années »accompagné d'une note qui témoigne d'une extrême confiance, puisqu'elle rend le destinataire dépositaire de ses archives "en cas d'événement imprévu ${ }^{30}$ "!

\footnotetext{
Vous êtes le premier à lire la 1ère esquisse de ce roman de 2000 pages, provisoirement intitulé «Les Quatre vérités».

Ci joint quelques squelettes de chapitres uniquement pour vous en donner une idée. Je vous les livre à titre confidentiel car ce roman est de nature à dresser contre moi les 3/4 de l'opinion dans l'état actuel de la littérature.

Vous jugerez sévèrement les oscillations de la forme, les naïvetés, l'imprécision des sujets. Mais songez que ce roman ne sera pas terminé avant plusieurs années et que je n'ai pu recopier ou faire taper qu'une infime partie des matériaux.

En cas d'événement imprévu, je déclare par la présente note confier à votre garde et à vos soins le présent manuscrit, ainsi que tous ceux qui sont actuellement disséminés à Alger (3, rue du Grand pieu, clos Salembier), dans ma chambre (277, Bld Péreire, XVIIème) et chez M. Chergui (50, rue du Four, Paris VIème).
}

21 Kateb est alors revenu à Paris et traîne dans les milieux littéraires et artistiques, où il croise de temps en temps Jean Sénac et Bertolt Brecht. Mais il faut bien vivre; pour cela, il s'inscrit "dans un centre de formation professionnelle à Montreuil, où on apprend la soudure $^{31} »$. Quelques jours après avoir déposé son manuscrit chez Audisio, il lui rend compte de ses premiers jours de formation : 
Cher Ami,

Un petit mot de triomphe ; après une journée d'atelier, c'est tout juste si l'électricité ne me paraît pas aussi grande que la poésie. J'ai commencé à rôder dans Montreuil et à manier la pince parallèle : de quoi enrichir et terminer le pire roman.

Il faut dire que mon optimisme prend quelque peu sa source chez Mme Berthelot. Très gracieusement reçu et aidé au-delà de mes espérances, j'ai l'impression que le problème de mes sœurs est en voie d'être résolu.

Maintenant, il me reste à profiter de toutes ces providences et à espérer une proche occasion de vous serrer la main.

Kateb Y32.

En août, non sans rappeler les différends idéologiques qui «auraient pu séparer l'homme mûr de l'adolescent, l'Européen du Berbère ", le jeune écrivain, annonçant par la même occasion un éloignement passager de Paris, se félicite de l'accueil reçu par son manuscrit auprès d'Audisio, malgré d'apparentes réserves de celui-ci :

Cher Ami,

Loin de moi l'idée d'exprimer ici tout ce qu'est pour moi votre lettre. Le premier éclair d'une compréhension que je n'avais pas espérée aussi vive, aussi amicale, malgré la vie d'homme qui eût pu séparer l'homme mûr de l'adolescent, l'Européen du Berbère. Bref.

Vous avez raison sur toute la ligne. À tel point que je décide d'abandonner tout projet de publication bien au-delà de toute limite - car l'art est long et je suis épuisé d'avoir passé un peu trop de nuits à griffonner sans nourriture, ni pour le corps ni pour l'esprit.

Je ne puis rester plus longtemps à Paris, bien que j'aurais voulu tenir jusqu'à votre retour et, qui sait, trouver un éditeur capable de financer quelque peu l'œuvre, s'il la trouve digne d'arriver à terme. Mais j'ai une occasion de travailler en respirant l'air de Grenoble pour un mois et je pars décidé à revenir.

Le plus affectueusement à vous,

Kateb Y33.

PS. Je vous écris les jours qui viennent de mon nouveau home.

De fait, une nouvelle et longue lettre ne tarde pas à suivre, portant comme adresse d'expédition : « Kateb chez Mme Toussaint - Percevalière - Sayssinet-les-Iles (Isère) » :

Cher Ami,

J'ai choisi pour vous écrire la gorge embuée d'une de ces montagnes dont Grenoble reçut sa brune fourmilière aux temps de la nation française en formation. Catholicisme et second Empire sont mes découvertes actuelles. Je m'émerveille non moins de mon retour parmi les cigales et j'apprends qu'un évêque d'ici a fait condamner les constructeurs d'un tunnel où les Nord-Africains périrent nombreux à demi salaire. Je passe au crible un flot d'idées - en majorité impraticables - sur les sources géologiques, juridiques, historiques, etc. que devrait boire une littérature à la mesure du siècle. Les amis qui m'hébergent ont une bibliothèque où je trouve au complet, pour la première fois et traduits, bien des auteurs escamotés jusqu'ici pour moi, de l'Antiquité à Buffon. Je m'instruis donc, sans me défendre cependant d'une grande impatience de revoir Paris, pour y terminer le roman, bien qu'un retour me soit devenu pour ainsi dire interdit, s'il faut songer à l'hiver, à la dèche, aux charges familiales. Je me vois en définitive coupé de toutes mes bases, au moins jusqu'aux vendanges, à moins que... Et vos vacances? J'espère que vos ennuis sont atténués et que j'aurai bientôt quelques mots de vous.

Affectueusement,

Kateb $Y^{34}$.

Dans une page manuscrite non datée - mais que la chronologie nous commande de placer à la fin de cet été ou au début de l'automne 52 -, Kateb indique à Audisio qu'il a 
profondément corrigé, et surtout classé et resserré, l'amas de textes qu'il lui avait adressé au printemps :

Cher Ami,

Enfin une forme est née aux travaux délaissés depuis presque trois ans. Votre encouragement a fait beaucoup. Si vous pouviez tout lire en me disant votre pensée, et si possible dans le détail, vous me rendriez très heureux. Le travail ci-joint peut être vu de deux façons :

$1^{\circ}$ un roman dont le titre reste à trouver

$2^{\circ}$ une série de nouvelles intitulées NOUVELLES D'OUTREMER

Dans un cas comme dans l'autre, la chronologie est la suivante :

I Deux têtards

II L'âme cet amour perdu

III Perdition de Mourad

IV Marchand de rêves

V Omar en France

VI Vue aérienne

Je crois qu'allonger la sauce serait nuisible.

Reste la question douloureuse de la publication. Je me suis tant fermé de portes ces temps-ci que je ne vois pas d'éditeur ni de revue capable de s'y intéresser (il est vrai que je ne lis ni ne sors pour ainsi dire plus). Or la situation est critique. Le travail littéraire m'a complètement repris et dominé, si bien que je ne peux plus retourner aux tâches manuelles (d'ailleurs difficiles et problématiques à trouver). Bref, j'attends de vous des critiques, des conseils, et une sage orientation.

Affectueusement.

Kateb $Y^{35}$

25 À la lecture de ces pages, la réaction d'Audisio est immédiate : il se trouve que son ami Guirao vient de créer à Oran la revue Simoun ${ }^{36}$, et qu'il a besoin de textes pour un numéro spécial consacré aux Lettres algériennes qu'il compte publier à Noël ${ }^{37}$. Il a donc sollicité Audisio - et il est probable que c'est lui qui, à défaut du texte introductif espéré par Guirao, a fait parvenir à la revue, peut-être sans en référer préalablement à l'auteur, un extrait de « Marchand de rêves ", l'un des textes du recueil (fig. 3 et 4). 
Fig. 3 : Extrait manuscrit du texte "Marchand de rêves "

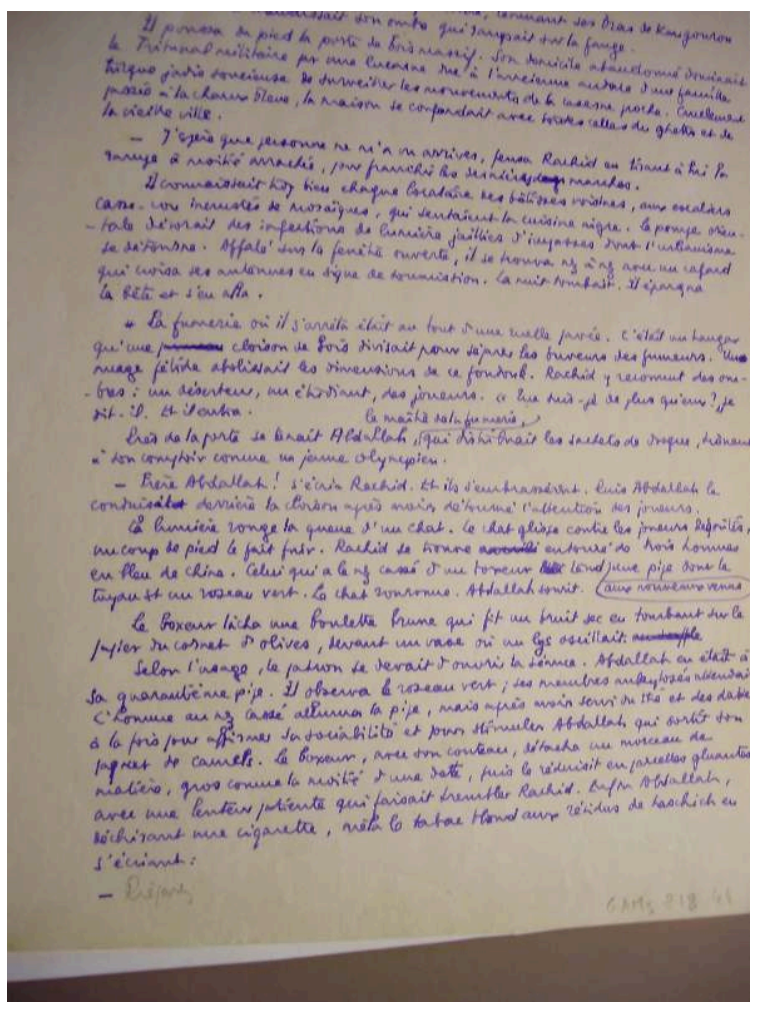

Fonds Audisio, Bibliothèque de l'Alcazar, Marseille

Fig. 4 : Extrait tapuscrit du texte "Marchand de rêves "

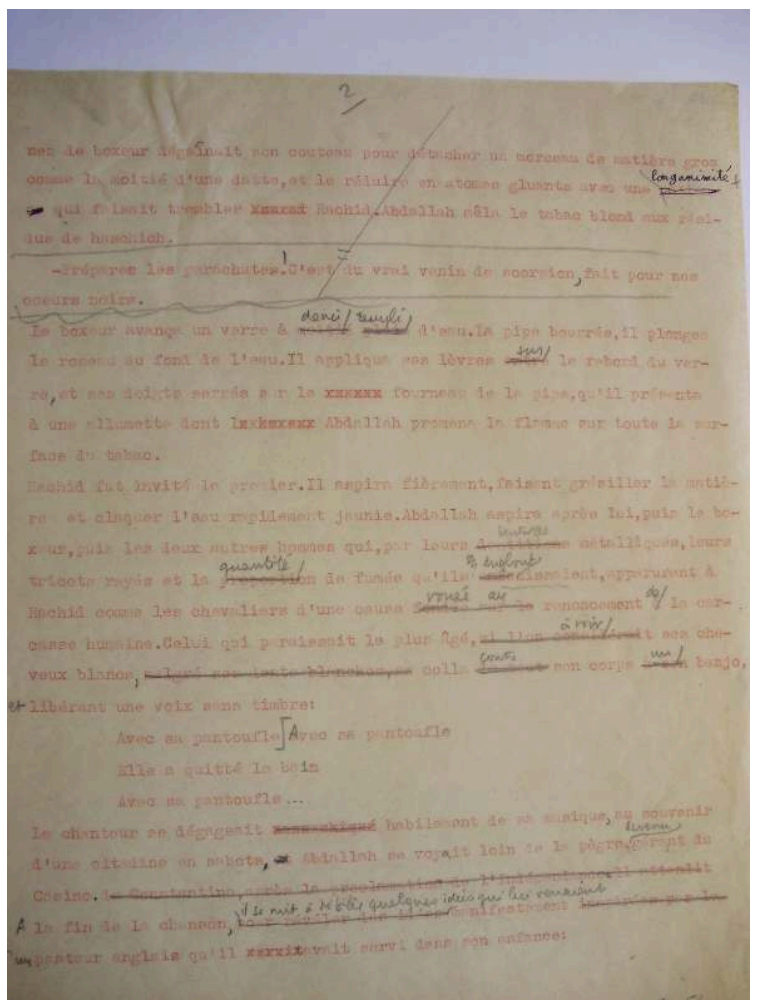

Fonds Audisio, Bibliothèque de l'Alcazar, Marseille 


\section{publication.}

Cher ami,

Je suis honteux du travail que je vous ai étourdiment donné à propos du texte pour

"Simoun". J'aurais dû préciser que, des coupures s'imposant, se trouvait posé un important problème de signification. Or un extrait de "Marchand de rêves", présenté comme une nouvelle, a une signification générale qui est en contradiction fondamentale avec le sens de mon œuvre qui se veut progressiste, alors que l'extrait en question peut paraitre une apologie ou tout au moins une idéalisation du milieu fumeurs : et cela, après un an de silence, peut donner à ma position actuelle une apparence de confusion que je veux éviter à tout prix (vous connaissez mon problème et vous me comprenez). J'ai été imbécile de ne pas prévoir cet aspect de la question lorsque nous en parlâmes.

S'il n'est pas trop tard pour "Simoun", il conviendrait peut-être d'envoyer "L'âme, cet amour perdu" ou "Un émigré", mais uniquement si vous avez le temps de le faire. Sans quoi, ce ne serait qu'une impossibilité secondaire, car vous recevrez ces jours-ci mon roman revu et augmenté de cent pages, d'après vos justes critiques.

Vives excuses et plus que jamais à vous,

Kateb Y ${ }^{38}$.

Finalement, c'est sous la forme de deux volumes que cette anthologie des Lettres algériennes, plus riche encore que ne l'espérait Guirao, paraitra l'année suivante ${ }^{39}$, avec dans le second un texte de Kateb n'est aucun de ceux proposés à Audisio, mais «À l'instar d'un match sanglant ${ }^{40} »$, qui sera plus tard intégré au Polygone étoilé. Ajoutons qu'un autre texte de Kateb paraîtra dans un numéro suivant de la revue, il s'agit de "L'Ancêtre et le têtard ", sans doute tiré de " Deux têtards ", dans le n 10.

En ce même automne 1952, Gabriel Audisio entreprend de soumettre le recueil de nouvelles qui lui a été confié par Kateb à la critique de son ami Roblès, qui vient d'intégrer l'équipe éditoriale des éditions du Seuil, où il a créé la collection "Méditerranée ", laquelle commence à publier des auteurs maghrébins de langue française :

Mon cher Roblès,

J'espère, sans en être sûr, que tu n'as pas fait un voyage de retour trop secoué par les flots...

Voici ce qui m'amène. J'ai enfin revu Kateb. Il est d'accord pour que je te soumette un manuscrit, "Nouvelles d'Outremer", dans l'état actuel, étant entendu que pendant 1 ou 2 mois il va s'appliquer à faire les ajouts et corrections que je lui ai suggérés dans une note dont tu trouveras ci-joint une copie et sur laquelle il est d'accord dans l'ensemble.

L'essentiel serait que tu me dises aussi vite que possible si cet "avant livre" t'inspire confiance. Moi, j'ai confiance: après avoir lu le Dib (très bien), le Feraoun et feuilleté le Mammeri ${ }^{41}$, j'estime que Kateb a ses chances : il a lui aussi quelque chose à dire et il le dit à sa façon. Dis-moi également si tu partages mon avis sur les retouches que je lui ai conseillées.

Enfin, et cela est plus délicat... je crois que Kateb est dans une situation matérielle très difficile. Il faudrait qu'il puisse "tenir" un peu, au moins jusqu'à la mise au point de son livre. Pense-tu - si dès à présent le Seuil s'intéressait à lui - qu'il serait possible de le faire aider de quelques subsides par anticipation?

$\mathrm{Tu}$ seras gentil de me dire ce que tu penses de tout cela. [...]

A toi, bien affectueusement. Mes hommages amicaux à ta femme.

Audisio $^{42}$

La lettre est suivie de deux pages de notes dactylographiées, pompeusement intitulées « Note de lecture »: 


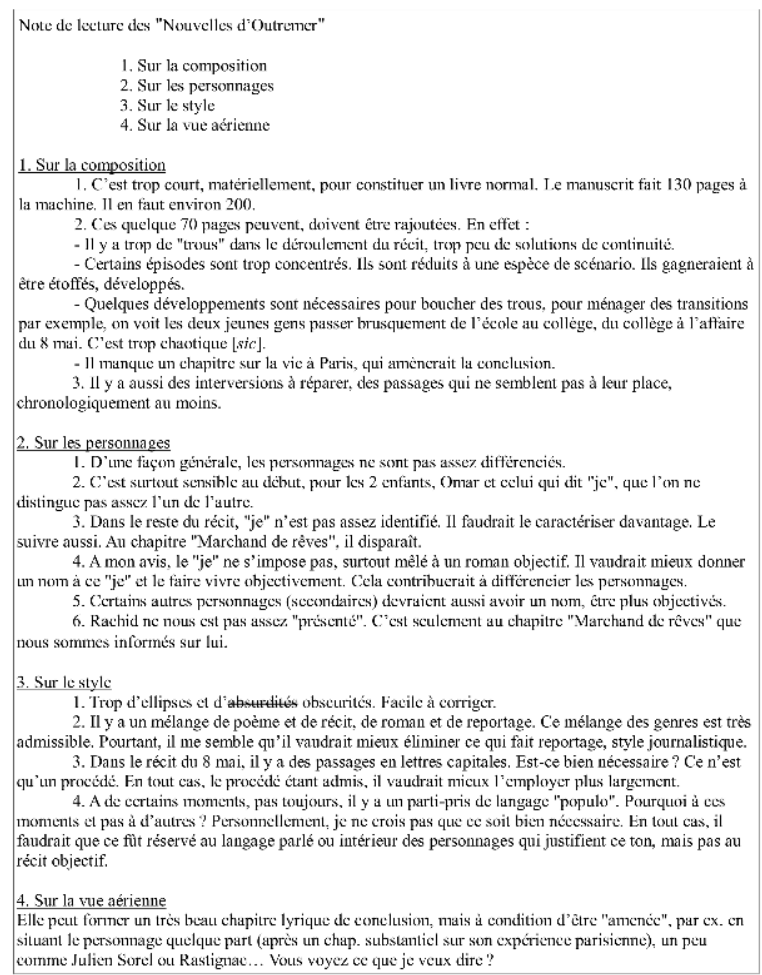

La réponse de Roblès ne tarde guère, franche et sévère :

Cher Audisio,

Je me suis jeté sur le manuscrit de Kateb avec l'appétit (et la sympathie) que tu devines.

Mais je ne suis pas de ton avis. Je ne lui fais pas confiance. Kateb est doué, c'est certain, mais le texte qu'il présente semble traduire une complète impuissance à adopter et à conserver un ton, à composer, à donner au livre une architecture.

L'ensemble est très décevant. Ce ne sont que matériaux bruts et tu comprendras que je ne puisse m'engager. Un menuisier peut avec de belles planches se proposer de fabriquer une armoire. Vais-je la payer d'avance, l'acheter sans savoir si elle sera belle, à mon goût, si elle me plaira et me conviendra?

Tes remarques sur la compo, les perso et le style sont toutes très justes. Mais elles sont très graves aussi. Après ces critiques (que je prends à mon compte), quels mérites reste-t-il au manuscrit? Peu de chose.

Il faut surtout conseiller à Kateb de maîtriser sa fougue, et qu'écrire un livre est une aventure sérieuse, à laquelle il semble mal préparé. Je n'ai vu dans ces 150 feuillets que des notations rapides, une désinvolture navrante, des dons gâchés.

Je te renvoie le manuscrit par ce même courrier. Naturellement, je suis prêt à lire la seconde version lorsqu'elle sera achevée. Mais je reste sceptique.

Bien affectueusement à toi

Roblès ${ }^{43}$ est-ce une autre filière qui a conduit des poèmes consacrés à Nedjma dans Le Mercure de France et Esprit à Nuit et jour ${ }^{44}$, puis à Nedjma?

Toujours est-il que le roman paraîtra bien aux Éditions du Seuil quelques années plus tard: mis en fabrication le 7 mars, il sera publié en mai 1956. Pas par Roblès cependant, ni dans sa collection "Méditerranée ", comme on le croit trop souvent ${ }^{45}$, mais par un autre biais : à partir de 1953, Kateb s'est rapproché de la revue Esprit, très proche de la maison d'édition; il y publie un portrait de $\mathrm{Nedjma}^{46}$, noyau du roman à venir, puis, en 
deux livraisons, sa pièce Le Cadavre encerclée ${ }^{47}$, qui le fera enfin connaître ; c'est là qu'il va croiser deux autres intellectuels, plus jeunes, plus militants, qui vont compter pour beaucoup dans la mise en forme définitive de son œuvre : Jean-Marie Serreau, pour le théâtre, et, pour l'œuvre romanesque, Francis Jeanson, lui-même déjà marginal dans cette maison d'édition qu'il va bientôt quitter.

Avec leur aide, commence alors un autre travail sur le texte, d'autres lectures et d'autres négociations... Kateb Yacine est enfin entré en littérature.

\section{NOTES}

1. Lettre inédite à G. Audisio : «Paris, avril 1947.// J'ai enfin classé mes papiers et j'ai groupé - bien plus que choisi - quelques pièces que je vous soumets.// Le souvenir de votre parfaite courtoisie à mon égard et la bienveillance qu'on devine en vous me laissent espérer que vous ne les détesterez pas trop.// [...] Kateb Yacine - 204 rue du Château des rentiers - Paris XIIIème. »

2. L'Office algérien d'action économique et touristique (OFALAC) comportait en son sein un organe spécialisé dans le domaine de l'information, le service algérien d'information et de presse, dont Gabriel Audisio (1900-1978), jusqu'alors affecté à l'office de l'aide aux Nord-Africains, avait été nommé directeur par un arrêté du 15 avril 1945. Par un arrêté du 21 janvier 1947, cet organe est rattaché à l'office administratif du Gouvernement général de l'Algérie à Paris, où Audisio s'installe donc. Le service algérien de presse et d'information réalisait une revue quotidienne de la presse parisienne pour le gouverneur général, se chargeait de la diffusion des documents produits par le service de l'information d'Alger et s'occupait de relations publiques et de l'organisation de manifestations diverses. Au fil du développement de la guerre d'Algérie, le caractère stratégique de l'information devient de plus en plus clair pour les services chargés des affaires algériennes. L'administration centrale souhaite alors non seulement être tenue informée de l'évolution de l'opinion publique, mais aussi se doter de moyens propres à l'influencer. Par l'arrêté du 28 juin 1958, l'Office administratif de l'Algérie est rattaché au secrétariat général auprès du Premier ministre pour les affaires algériennes. Progressivement, les services de l'Office sont incorporés aux services du secrétariat général. En 1958, Gabriel Audisio est nommé conseiller culturel du secrétaire général pour les affaires algériennes.

3. Le fonds Audisio de la bibliothèque de l'Alcazar à Marseille conserve une soixantaine de lettres de Kateb (cote GAM3, 2181 à 60). La première, une lettre de vœux manuscrite recto-verso au format A4, est datée "Lafayette 1er janvier 1947 », mais son ton lyrique et familier laisse entendre que les deux hommes sont déjà en relation depuis quelque temps: Cher Ami,// Je vous souhaite, ainsi qu'à votre famille, la meilleure année. Je ne sais si vous connaissez Lafayette. C'est un ahurissant village kabyle, illuminé, pauvre, froid, petit - j'y ai mes vieux parents très malades dans une maison toute saisie par le givre et le soleil, toit creux sentant les briques remplies de cafards et le feu éteint. La neige lève à la lumière, des ouvriers terrassiers chassent la boue en maugréant. Il y a des différents terribles entre notre vieux chat noir (un sans appétit à vous faire pleurer) et certain coq habité du démon, acheté dans un douar, cauteleux, sautillant, rêveur, sentant la mort prochaine et qui lève un bec excédé, sus au chat, ce qui vraiment nous transporte tous. Le mendiant aveugle Sghir, ami élégant et malchanceux, philosophe, célibataire indulgent, dit que ce sont deux âmes ennemies et que cette hostilité, loin de l'étonner, l'édifie. Hegel et même M. Sartre ne le désavoueraient pas. Moi, je vous serre vigoureusement les mains en attendant de vos nouvelles. Kateb $Y$. 
4. À M. André Walter. « Paris, le 13 mai 1947 ».

5. Kateb Yacine, Paris, Seghers, coll. « Poètes d'aujourd'hui », p. 15-16.

6. Publié en 1948 à Alger par l'imprimerie En-Nadha, ce petit texte, qui figure dans le fonds Patrimoine méditerranéen de l'université Montpellier 3, a été réédité en 1989 par les éditions nationales ENAL.

7. Voir notamment l'article d'Abdellali Merdaci, «Kateb Yacine avant Nedjma, les imprescriptibles médiations d'une entrée dans la littérature ", dans Le Soir d'Algérie, samedi 30 octobre 2010. En ligne: http://www.lesoirdalgerie.com/articles/2010/10/30/article.php? sid $=108055 \&$ cid $=16$

8. C'est à Afifa Bererhi que l'on doit le point le plus complet sur ces rencontres de l'hiver 1947-1948, initiées par le service des mouvements de jeunesse et d'éducation populaire, rattaché au Gouvernement général. Voir son article « 1948. Les Rencontres de Sidi Madani. Projet culturel et enjeux politiques» dans l'ouvrage collectif Défis démocratiques et affirmation nationale. Algérie, 1900-1962, Alger, Chihab éditions, 2016.

9. Voir notamment lettre du 13 mai 1947 de Kateb Yacine à André Walter et celle de janvier 1948 à Gabriel Audisio, reproduite ci-dessous.

10. Sur la collaboration de Kateb à cette revue, voir notre article " Genèse de Forge et Terrasses » dans le précédent numéro de Continents manuscrits. En ligne: http://journals.openedition.org/ coma/938

11. Dans la lettre à Gabriel Audisio d'avril 1947 déjà citée, Kateb précise : «je dois vous dire que je suis irrémédiablement brouillé avec M. Amrouche, ce qui rend les contacts plutôt gênants avec l'école Algérienne. Aussi est-ce à vous seul que je m'adresse.// je vous serre très fort les mains.// Kateb Yacine 204 rue du Château des rentiers - Paris XIIIème ». L'allusion à « l'école algérienne » et à une rupture avec Amrouche, alors tout-puissant directeur littéraire chez Charlot et rédacteur en chef de la revue L'Arche, est preuve que Kateb aspire déjà à une insertion dans le champ littéraire de la colonie.

12. Il s'agit du poème qui clôt le recueil Soliloques. On trouve dans la version de Forge une seule modification, minime mais intéressante, suggérée par Roblès pour éviter la difficulté phonétique constituée par la répétition de la labio-dentale [f] : «Où dormait mon front ", au lieu de "Où dormait mon front fier ».

13. Préfacière de Soliloques.

14. Le texte Ravissements de la fête, comme la plupart de ceux mentionnés dans cet article, n'apparaît pas dans la Bibliographie Kateb Yacine dirigée par Charles Bonn (L'Harmattan, coll. "Études littéraires maghrébines ", n 11, 1997).

15. Lettre manuscrite datée du 26 avril 48 et signée «Kateb Y. - 17 rue des Chapeliers Marseille ». Au verso, trois lignes dactylographiées barrées: "Quand je me propose de faire de grands efforts et que je trouve sur mon chemin une trop grande résistance, je fais de longs voyages à travers l'Algérie avec l'espoir de comprendre comment un peuple si compliqué arrive à vivre. "

16. Dans les $n^{\text {os }} 12$, décembre 1954 , et 1 , janvier 1955. On rappellera que c'est après lecture dans Esprit de cette "tragédie en 3 actes " que Jean-Marie Serreau vint frapper à la porte de Kateb Yacine, comme il l'a raconté (entretien avec Moussa Abadi, RFI, émission Images et visages du théâtre d'aujourd'hui du 22 février 1967, archives IMEC).

17. Voir « Combat » des 4, 17, 18, 24 et 31 août 1948 [note de K.Y.].

18. "Monsieur Audisio prétendait contre moi que durant l'occupation, au moment où les relations avec la métropole étaient coupées, le Ministère de l'Intérieur allouait des mensualités aux étudiants algériens privés de soutien familial. Ceux que j'ai interrogés n'ont rien touché, et ce sont bien les commerçants algériens qui les soutenaient par des collectes " [note de K.Y.].

19. Le brouillon de cette lettre, du 15 novembre [1948], référencée $\mathrm{GA} / \mathrm{SB} / \mathrm{n}^{\circ} 2794$, figure également dans le fonds Audisio de la bibliothèque de l'Alcazar (pelure A4 dactylographiée). 
20. Reproche adressé par Audisio à Kateb d'être allé enquêter sur la condition des étudiants algériens sur témoignages plutôt que d'après les statistiques ministérielles. Lettre du 15 novembre, ibid. : "Quant à la question des étudiants, je vous avais indiqué la source officielle où vous pouviez vous renseigner notamment sur ce qui a été fait et sur ce qui n'a pas été fait. Avez-vous été à cette source?[...]»

21. Lettre manuscrite, format A4; en-tête: "Front national démocratique algérien" - $124 \mathrm{Bl}$ Voltaire - Paris XIème (fig. 2).

22. Kateb Yacine : "À l'époque, c'était le Parti communiste algérien qui voulait créer le Front national démocratique (une des préfigurations $d u F L N$ ) », entretien avec Tassadit Yacine, op. cit.

23. Texte inédit d'une conférence prononcée devant la commission culturelle du Front national démocratique algérien, le 20 novembre 1948. Cinq feuillets A4 dactylographiés avec de nombreux rajouts manuscrits [cote GAM3 218 - 8 à 12].

24. Au début $\mathrm{du} \mathrm{xx}^{\mathrm{e}}$ siècle, l'économiste anglais Hobson avouait: "Le Christianisme, qui a pris rapidement pied dans un petit nombre de grands empires, dont chacun possède une série de colonies peu civilisées et pays assujettis, apparaît à beaucoup comme le développement logique des tendances modernes, développement qui donne le plus d'espoir en une paix durable (!) fondée sur l'inter-impérialisme» [note manuscrite de K.Y.].

25. "Seuls étaient préservés les féodaux algériens dont le "prestige" servait à l'occupant» [note manuscrite de K.Y.].

26. Liberté, 29 juillet 1950.

27. Lettre manuscrite, format A4, «Lafayette, mars 1949 ». Le pluriel «Chers amis » laisse à penser à une demande collective des services d'information du Gouvernement général, que dirigeait alors Audisio.

28. Dans une lettre datée "Alger, 8/12/51", Kateb dit à son correspondant avoir "beaucoup regretté de n'avoir pu [le] joindre cet été à Paris où [il a] terminé de laborieuses vacances. Ce n'est que partie remise puisque je reviens cet hiver avec un roman terminé que je vous enverrai dès qu'une dactylo me sera devenue abordable ».

29. Lettre non datée [septembre ou octobre 1951].

30. Note datée du 21 mars 1952. Quelques mois plus tard, en juillet, le poète joindra à ce dépôt initial «deux cahiers formant chapitre central du roman dont vous avez quelques parties. je vous téléphonerai comme convenu dans une semaine " (petit billet manuscrit, daté au crayon « juillet $52 »)$.

31. C'est ainsi que Kateb Yacine, ajoutant que "cela n'allait pas [parce qu'il était] trop nerveux", présente cette expérience et son rapide abandon dans un entretien accordé à Nadia Tazi, recueilli dans Le Poète comme un boxeur, Paris, Le Seuil, 1994, p. 24.

32. Lettre manuscrite, format A4, datée « 24 mars 1952 ».

33. Lettre manuscrite, format $A 4$, datée « 17 août 52 ». Après la mort de son père en 1950 , Kateb avait à charge sa mère et ses deux sœurs.

34. Lettre manuscrite, format $\mathrm{A} 4$, datée « $22 / 8 / 52$ ». Audisio répondra à ces deux lettres le 4 septembre, en regrettant de n'avoir pas su plus tôt que Kateb était si près de Grenoble : "Quel dommage, je viens de passer plus de 24 heures à Grenoble où j'étais en panne de voiture! Si votre lettre m'avait suivi, j'aurais eu des chances de vous y rencontrer ", et en formant des vœux pour rencontrer son correspondant à Paris " pour vous dire exactement tout ce que je pense de votre manuscrit? Sinon, dès que je le pourrai, je vous ferai un petit compte rendu, dans l'espoir de vous être utile [...]. »

35. Une page manuscrite A4 non datée.

36. Sur cette revue et son fondateur, voir Jean-Michel Guirao et la revue SIMOUN, revue CELAAN, Centre d'études des littératures et des arts d'Afrique du Nord, vol X, No. 1 \& 2, Spring 2012.

37. Voir notamment la lettre de Guirao à Audisio du 25 octobre 1952 : «Ce numéro spécial prend une belle allure déjà. Nous avons la collaboration de Rosfelder, Lentin, E. Dermenghem, El Boudali Safir, Mohammed Dib, Mouloud Feraoun, Marcel Moussy, Millecam, E. Roblès (une pièce en 1 acte inédite). J'ai écrit à R. Celly, A. Camus. Cela ne s'arrêtera pas là. Si toutefois vous aviez l'occasion de demander leur 
collaboration à ce numéro à quelques autres écrivains (desquels je n'aurais l'adresse ou que j'oublie), vous me rendriez service. [...]»

38. Lettre manuscrite, format A4, datée « Paris, le 10 novembre 1952 ».

39. $\mathrm{N}^{\circ}$ 6-7, 10 février et 8,13 avril 1953.

40. «Écrivains algérien ", Simoun, $\mathrm{n}^{\circ}$ 8, avril 1953, p. 62-67.

41. Audisio fait ici allusion aux tout premiers volumes publiés par Roblès dans sa nouvelle collection : La Grande Maison de Mohammed Dib (1952), La Terre et le sang de Mouloud Feraoun (1953). En revanche, ce n'est pas au Seuil, mais chez Plon, que seront publiés les premiers romans de Mouloud Mammeri.

42. Lettre dactylographiée, format A4, du 7 octobre 1952.

43. Lettre dactylographiée, format A5, datée « 15 octobre 52 ».

44. Titre initial figurant dans le dossier de fabrication conservé à l'IMEC, sous la cote SEL2.S2.B574.D5, auquel Jeanson semble avoir préféré celui de Nedjma.

45. Notamment Jean-Yves Mollier, «Un essai de dépassement des communautés partisanes : la collection «Méditerranée» au Seuil pendant la guerre d'Algérie (1945-1962) », Cahiers de la Méditerranée [En ligne], 85 |2012, mis en ligne le 14 juin 2013, consulté le 18 décembre 2017 : http://journals.openedition.org/cdlm/6657

46. Esprit, décembre 1953.

47. Dans les numéros de décembre 1954 et janvier 1955. Jean-Marie Serreau a lui-même raconté comment il vint frapper à la porte de Kateb Yacine après avoir lu dans Esprit cette «tragédie en trois actes ».

\section{RÉSUMÉS}

Entre absence de documents et mythes, parfois véhiculés par l'auteur lui-même, les conditions de la genèse de Nedjma (1956), roman majeur de la littérature maghrébine de langue française, demeurent obscures, plus de soixante ans après sa publication aux éditions du Seuil. Cet article, fondé sur des correspondances inédites des années 1946-1952, vise à faire le point sur cette énigme éditoriale - elle marque l'entrée en littérature de Kateb Yacine - et démontre notamment pourquoi cet écrivain du Seuil, souvent présenté comme un des auteurs phares de la collection «Méditerranée », ne publia pourtant jamais dans cette collection.

\section{INDEX}

Mots-clés : Yacine Kateb, Gabriel Audisio, littérature maghrébine de langue française, éditions du Seuil, Méditerranée

\section{AUTEUR \\ GUY DUGAS}

Chercheur associé à l'équipe Manuscrits francophones, ITEM (CNRS-ENS) 